\title{
Experimental Investigation of Neutral-Neutral Reactions and Energy Transfer at Low Temperatures
}

\author{
Ian R. Sims \\ Laboratoire PALMS - UMR CNRS-Université 6627, \\ Equipe 'Astrochimie Expérimentale', Campus de Beaulieu, Bât. 11C, \\ Université de Rennes 1, 35042 RENNES Cedex, France
}

\begin{abstract}
Over the last few years, we have applied the CRESU (Reaction Kinetics in Uniform Supersonic Flow) technique to the study of neutral-neutral reactions and energy transfer processes in the gas phase. This has enabled the rates of a wide range of reactions between electrically neutral species to be measured down to temperatures as low as $\sim 10 \mathrm{~K}$. These results have generated significant interest amongst theoretical chemists, and especially amongst astrochemists. Our measurements of low-temperature rate coefficients have had a significant impact on the models used to simulate the chemistry of dense interstellar clouds. In this article the motivations for the experimental study of reaction kinetics at low temperatures are described, and a detailed description of the CRESU technique is given, followed by a brief overview of the results obtained in Rennes and Birmingham. Four recent low-temperature kinetic studies are then described: the reaction $\mathrm{O}+\mathrm{OH}$ and the interstellar oxygen problem, reactions of the $\mathrm{C}_{2}$ radical, energy transfer in collisions of $\left.\mathrm{C}^{3} \mathrm{P}_{J}\right)$ with $\mathrm{He}$ and $\mathrm{H}_{2}$, and preliminary results on the reaction kinetics of the $\mathrm{C}_{4} \mathrm{H}$ radical.
\end{abstract}

Keywords. astrochemistry — ISM: molecules — methods: experimental — molecular processes

\section{Neutral-Neutral Reactions and Energy Transfer at Low Temperatures}

The temperature dependence of rate coefficients $k(T)$ for many reactions between electrically neutral species are found to obey, to a good approximation, the Arrhenius expression:

$$
k(T)=A \exp \left(-E_{a} / R T\right)
$$

with $E_{a}$, the activation energy, which is closely related to the barrier to reaction along the minimum energy path. This expression, first proposed by Arrhenius in 1889, has enjoyed such widespread success that 'Arrhenius plots' (of $\ln k(T$ )against $1 / T$ ) have become the standard way of reporting kinetic data as a function of temperature.

The validity of the Arrhenius equation, however, is based on the idea of activation energy, and relies on the presence of an energetic barrier to reaction. Rates of barrierless reactions are not expected to display Arrhenius behaviour, and are good candidates for study at low temperatures. These include: ion-molecule reactions, radical-radical reactions, and even many radical-molecule reactions.

An example of such a barrierless reaction is that between the $\mathrm{CN}$ radical and the ethyne molecule $\mathrm{C}_{2} \mathrm{H}_{2}$, which was found by us to display a rapid rate constant that increases with decrease in temperature (Sims et al. 1993). Subsequent quantum chemical calculations by Woon \& Herbst (1997) have shown that the potential energy surface for this reaction has the form shown in Figure 1. 


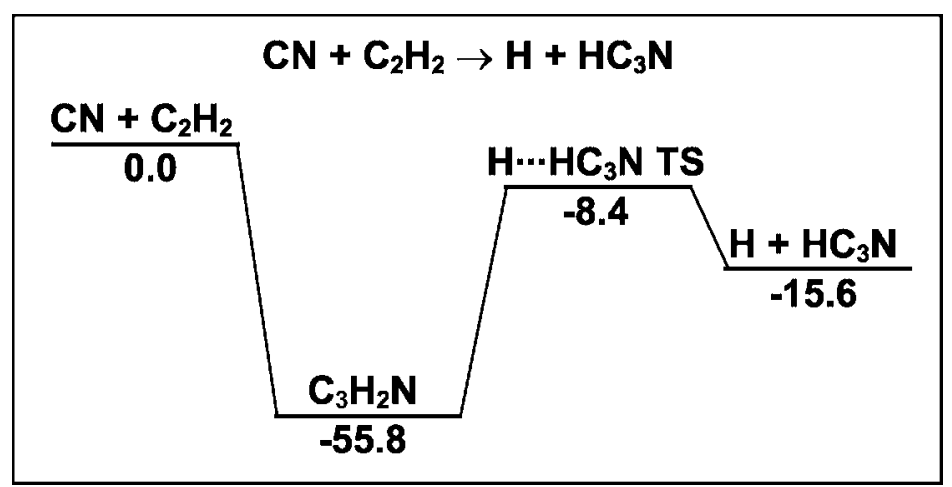

Figure 1. Minimum-energy potential pathway for the reaction of $\mathrm{CN}$ radicals with ethyne, as calculated by Woon \& Herbst (1997). The computed relative energies (kcal mol ${ }^{-1}$ ) listed for the stationary points (reactants, complex [energy minimum], transition state [TS; energy saddle point], and products) are given without zero-point energy corrections.

Prediction of rate coefficients at low temperatures for use in astrochemical models can be problematical. In the first place, extrapolation of rate data gathered at higher temperatures is not recommended as the form of temperature dependence may be complex. This is illustrated in Figure 2, which shows a sequence of Arrhenius plots for the reaction of $\mathrm{CN}$ radicals with ethane, $\mathrm{C}_{2} \mathrm{H}_{6}$.

While $a b$ initio quantum chemical calculations have progressed rapidly over the past decade, they cannot yet predict arbitrary neutral-neutral rate constants over wide ranges of temperature with the necessary degree of accuracy.

It is therefore apparent that accurate measurements of neutral-neutral rate constants are necessary down to temperatures as low as $10 \mathrm{~K}$ prevailing in dense interstellar clouds (ISCs). Furthermore, low temperature measurements of rate coefficients for collisional energy transfer - rotational excitation and de-excitation - are also sorely needed. These constitute some of the principal motivations for the experimental work described in this brief review.

\section{Techniques for Measuring Rate Coefficients at Low Temperatures: The CRESU Technique}

The CRESU (Cinétique de Réaction en Ecoulement Supersonique Uniforme, or Reaction Kinetics in Uniform Supersonic Flow) apparatus was devised by Rowe and co-workers (Rowe et al. 1984; Rowe \& Marquette 1987) initially for the study of ion-molecule reactions at extremely low temperatures. The strength of the technique lies in the nature of the isentropic expansion of gas through a Laval nozzle, which produces a flow of gas that is uniform in temperature, density and velocity, and which endures for several tens of centimetres and some hundreds of microseconds downstream of the nozzle exit. Frequent collisions occur during the controlled expansion within the nozzle and in the subsequent uniform region downstream where the gas density $\left(10^{16}-10^{17}\right.$ molecule $\left.\mathrm{cm}^{-3}\right)$ is relatively high. Consequently, thermal equilibrium is maintained at all times, in contrast to the situation in free jet/molecular beam environments. The expansion is slow enough to maintain thermal equilibrium, but rapid enough that condensation is avoided (strongly supersaturated conditions prevail in the subsequent flow). A uniform, 'collimated' flow results at the exit of the nozzle. 


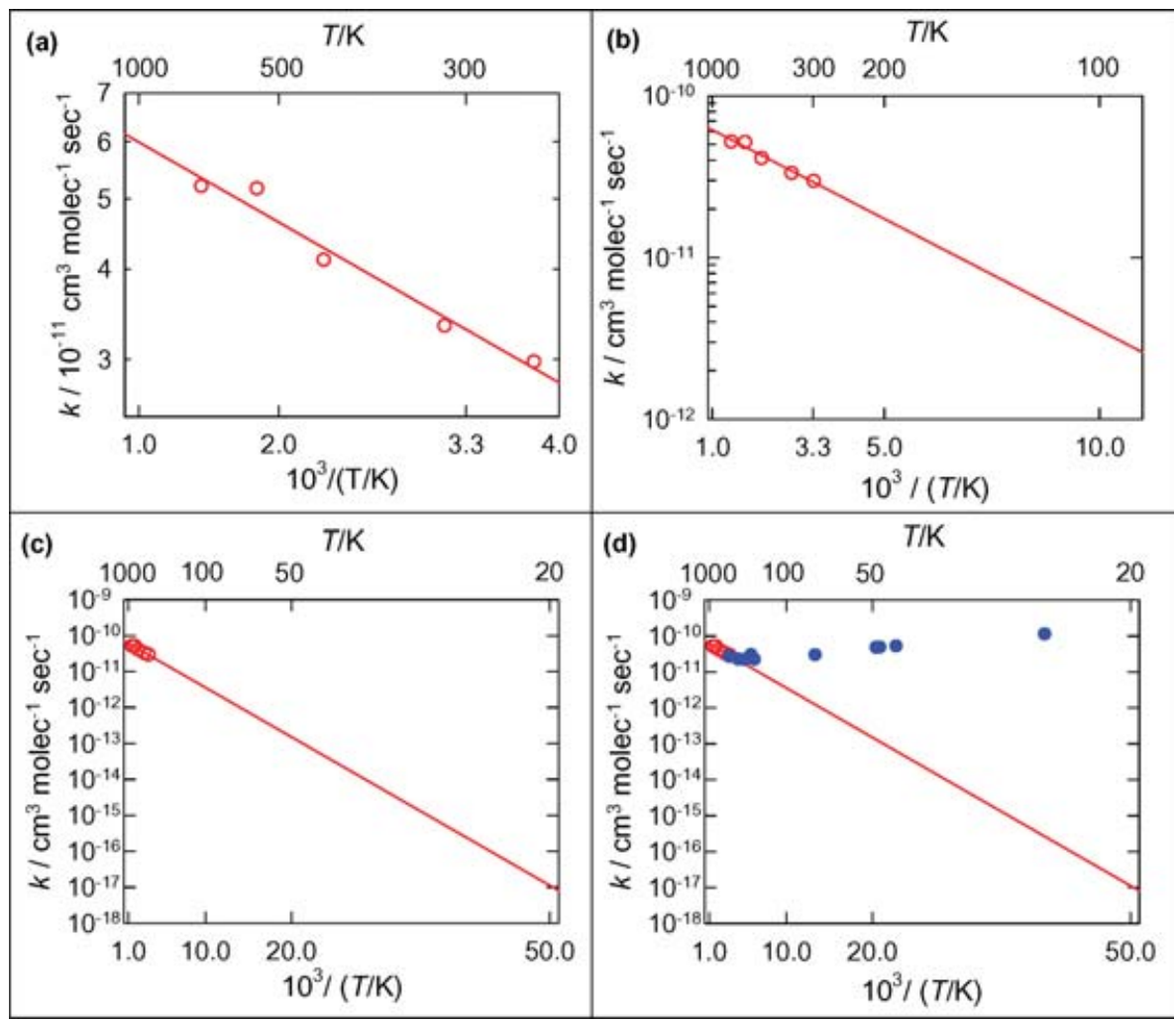

Figure 2. Rate constants measured for the reaction of $\mathrm{CN}$ radicals with ethane, $\mathrm{C}_{2} \mathrm{H}_{6}$, presented in the form of Arrhenius plots (Sims et al. 1993): (a) measurements made in heated or cooled cells (open circles) which fit well to an Arrhenius expression (solid line); (b) data extrapolated to $100 \mathrm{~K}(c)$ unjustifiably long extrapolation needed to reach $20 \mathrm{~K}$, where the predicted rate constant is vanishingly small; $(d)$ rate constants measured using supersonic flow techniques down to $25 \mathrm{~K}$ (solid circles), demonstrating the danger of extrapolating to low temperature the behaviour of rate constants at higher temperatures!

The CRESU technique provides an excellent environment in which to perform experiments on collisional processes at extremely low temperatures, not only between ionic and neutral species, but also between exclusively neutral species. This realisation, and the subsequent adaptation of the CRESU technique for the study of neutral-neutral reactions by the incorporation of the PLP-LIF technique, has enabled kinetic measurements on neutral-neutral reactions down to temperatures as low as $13 \mathrm{~K}$ (Bocherel et al. 1996; Sims et al. 1994).

A schematic diagram of the CRESU apparatus as used for the study of neutral-neutral reactions is shown in Figure 3. Photolysis and probe laser beams co-propagate through the throat of the Laval nozzle and out along the axis of the supersonic flow. The pulsed photolysis laser (or, sometimes, in the case of energy transfer studies, the excitation laser) creates a population of radicals by photodissociation of a precursor species introduced at low concentration into the gas flow. These radicals then collide with a co-reagent species also present in the flow, but in large excess with respect to the radical, whose concentration then decays according to (pseudo-)first-order kinetics, and is usually probed at set time delays by a second tunable laser which excites fluorescence (LIF) from the 


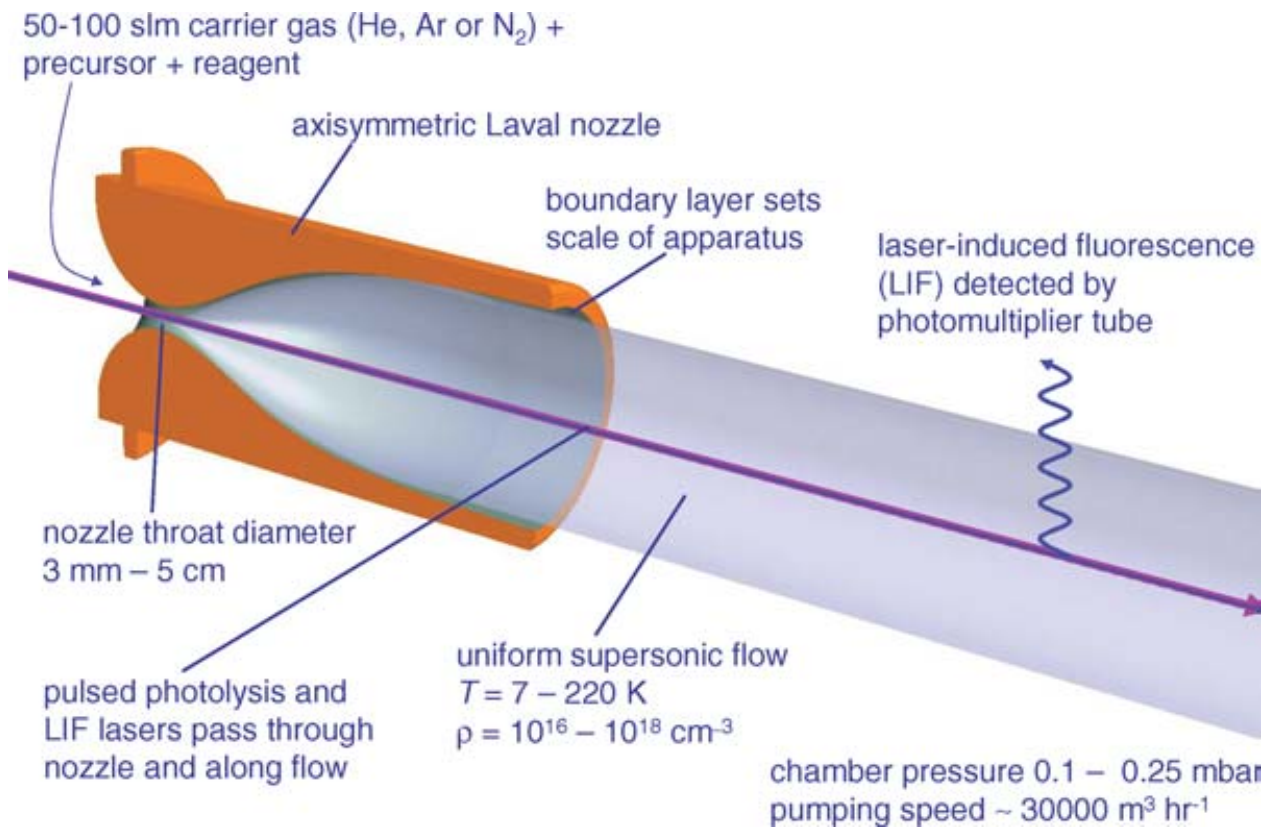

Figure 3. Schematic diagram of the CRESU apparatus depicting the Laval nozzle and supersonic flow, combined with pulsed laser photolysis - laser induced fluorescence (LIF) detection permitting the study of neutral-neutral reaction kinetics at extremely low temperatures.

radical species. The resultant exponential decay is fitted to yield a pseudo-first-order rate constant, and the experiment repeated at various co-reagent concentrations to yield a second-order plot, the gradient of which yields the rate constant of interest at the temperature of the supersonic flow. Full details of the technique may be found in Sims et al. (1994) and James et al. (1998). An example of the signal and results obtained for the reaction (vibrational relaxation) of $\mathrm{CH}(v=1)$ with $\mathrm{CO}$ may be seen in Figure 4.

The Rennes CRESU facilities currently comprise:

- One chamber dedicated to ion-molecule and electron attachment processes;

- Two chambers (Rennes/ex-Birmingham) dedicated to neutral chemistry (with room temperature or cooled reservoirs);

- One chamber with heated reservoir dedicated to very condensable species (PAHs); and

- One chamber dedicated to IR spectroscopy of aggregates.

\section{A Brief Survey of Past Results}

A surprising range of reactions studied in the CRESU apparatus have been found to remain rapid down to extremely low temperatures, including:

Radical-radical metathesis reactions, e.g. $\mathrm{CN}+\mathrm{O}_{2}$ (Sims et al. 1994$), \mathrm{C}\left({ }^{3} \mathrm{P}\right)+$ $\mathrm{O}_{2}$ (Chastaing et al. 2000);

Radical-unsaturated molecule reactions, e.g. $\mathrm{CN}+\mathrm{C}_{2} \mathrm{H}_{2}$ (Sims et al. 1993), $\mathrm{C}_{2} \mathrm{H}+$ $\mathrm{C}_{2} \mathrm{H}_{2}$ (Chastaing et al. 1998), $\mathrm{C}+\mathrm{C}_{2} \mathrm{H}_{2}, \mathrm{C}_{2} \mathrm{H}_{2}, \mathrm{C}_{3} \mathrm{H}_{4}$ (Chastaing et al. 2001);

Radical-saturated molecule reactions, e.g. $\mathrm{CH}+\mathrm{CH}_{4}$ (Canosa et al. 1997), $\mathrm{CN}+$ $\mathrm{C}_{2} \mathrm{H}_{6}$ (Sims et al. 1993). 

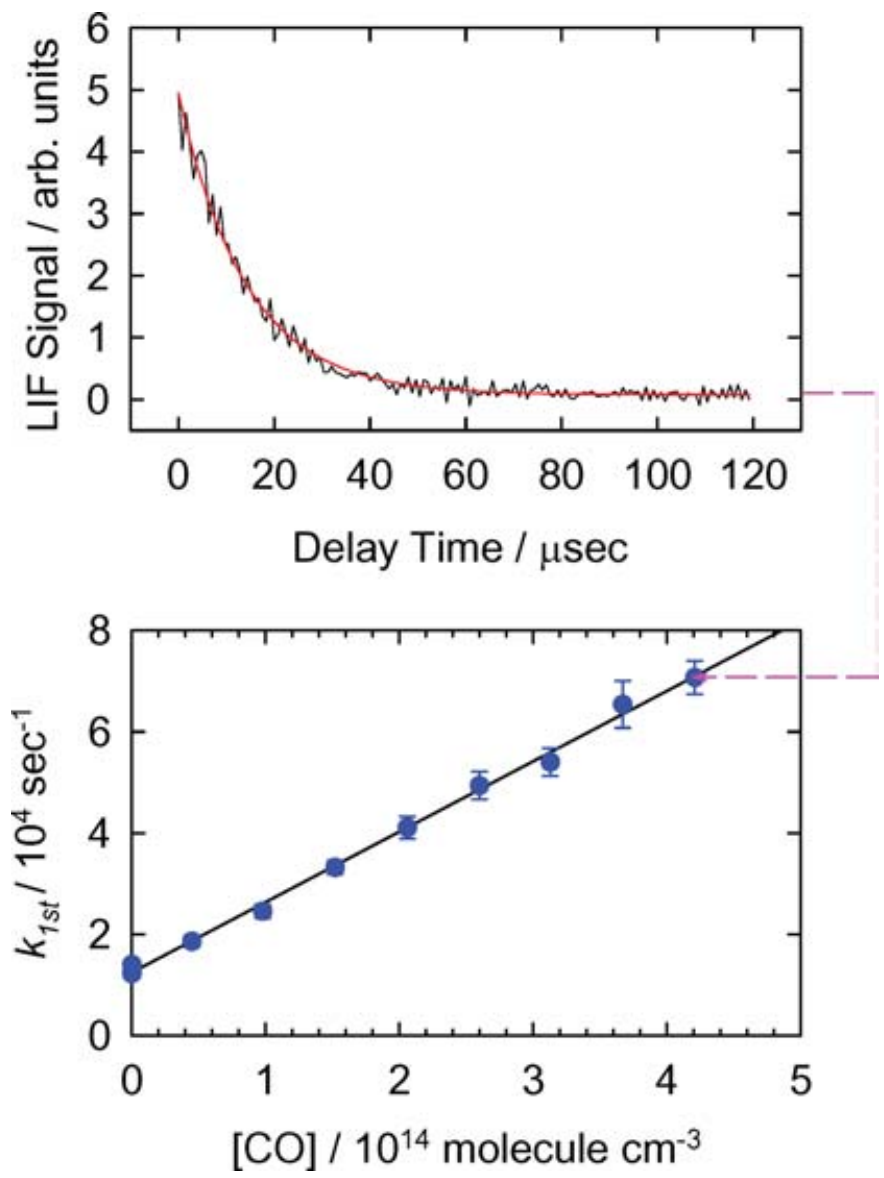

Figure 4. Upper panel: First-order decay of LIF signal from $\mathrm{CH}(v=1)$ in the presence of $4.2 \times 10^{14}$ molecule $\mathrm{cm}^{-3}$ of $\mathrm{CO}$ at $44 \mathrm{~K}$ in Ar, fitted to a single exponential decay. Lower panel: First-order decay constants for $\mathrm{CH}(v=1)$ at $44 \mathrm{~K}$ in $\mathrm{Ar}$ plotted against the concentration of $\mathrm{CO}$.

A number of reviews are available (Sims 1997; Sims \& Smith 1995; Smith \& Rowe 2000). These results have generated significant interest amongst theoretical chemists, and especially amongst scientists working in the area of astrochemistry. These measurements of low-temperature rate coefficients have had a significant impact on the models used to simulate the chemistry of dense ISCs, as has been recognised by leading astrochemical modellers such as Millar (Herbst et al. 1994) and Herbst (Herbst 2001; Terzieva \& Herbst 1998).

\section{Some Recent Studies}

\subsection{The $\mathrm{O}+\mathrm{OH}$ Reaction}

The reaction of ground state oxygen atoms with ground state $\mathrm{OH}$ radicals is of major interest from theoretical, experimental and astrochemical points of view. It is thought to be the main source of interstellar dioxygen:

$$
\mathrm{O}\left({ }^{3} \mathrm{P}\right)+\mathrm{OH}\left(X^{2} \Pi\right) \rightarrow \mathrm{O}_{2}\left({ }^{3} \Sigma\right)+H\left({ }^{1} \mathrm{~S}\right)
$$




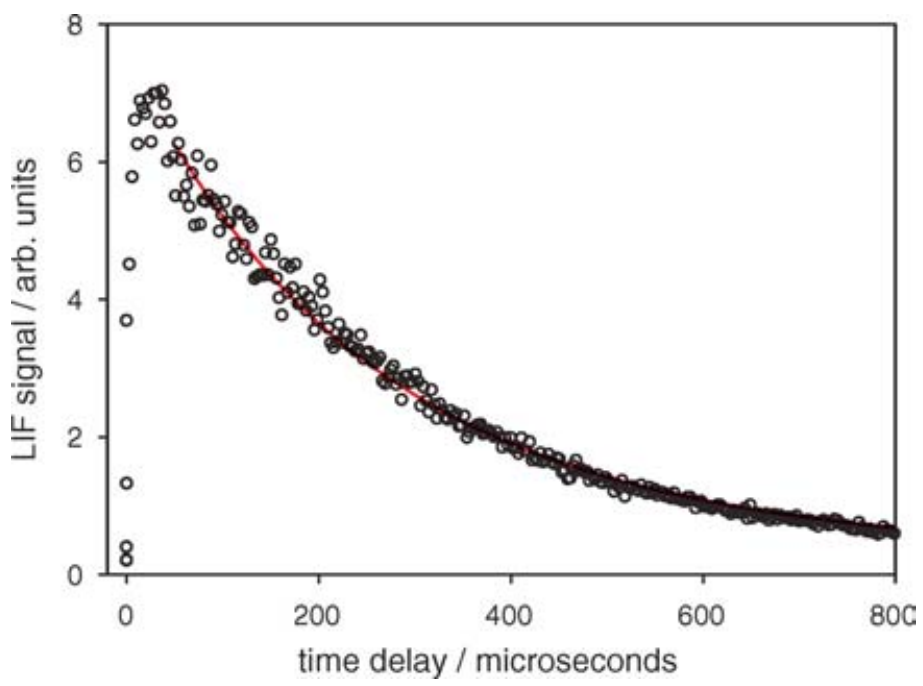

Figure 5. Decay of OH LIF signal in the presence of $\mathrm{O}\left({ }^{3} \mathrm{P}\right)$ atoms at $83 \mathrm{~K}$, fit to a single exponential decay.

However, successive attempts to detect interstellar $\mathrm{O}_{2}$ have pushed the upper limit for $\mathrm{O}_{2}$ abundance well below model predictions. A number of explanations have been advanced for this discrepancy, but clearly one important factor may be that the rate constant for the $\mathrm{O}+\mathrm{OH}$ reaction currently employed at low $(T<50 \mathrm{~K})$ temperatures is overestimated, as it is derived by extrapolation of measurements made at $160 \mathrm{~K}$ and above.

The $\mathrm{O}+\mathrm{OH}$ reaction has long been a challenging system both from a theoretical and an experimental point of view. The number of possible potential energy surfaces in this reaction between two open shell species renders theoretical calculations especially difficult. The usual techniques for the experimental measurement of gas-phase bimolecular reactions rely on the ability to achieve pseudo-first order conditions, requiring one of the reagents (usually the stable one) to be placed in a known excess of concentration, while the concentration of the other (usually radical) reagent is monitored in a relative but time resolved fashion by, e.g., laser induced fluorescence. For a reaction between two unstable species this obviously presents significant difficulties: the need to produce a significant concentration of an unstable species; and the requirement to measure or otherwise know this concentration. For reactions between atomic and molecular radicals, such as $\mathrm{O}+$ $\mathrm{OH}$, this has been achieved using a microwave discharge to produce the atomic radical in excess, and gas-phase titration to measure its concentration, coupled with, for example, pulsed photolysis and LIF on the molecular radical to measure the kinetics. For this reaction, such techniques have permitted the measurement of the rate constant down to temperatures as low as $160 \mathrm{~K}$ using cryogenic cooling (Smith \& Stewart 1994). However, adaptation of these techniques for use in the CRESU apparatus presents a number of major problems: firstly, as the mass flow rates are much higher than in a typical flow tube, higher power discharges are needed; secondly, the gas pressures before and after the Laval nozzle expansion are fixed by aerodynamical considerations, resulting in conditions which are non-ideal for the production and maintenance of high concentrations of atomic radicals; and finally, the usual gas titration techniques are not possible, or at least difficult in the supersonic flow. Solutions to these problems are currently being sought in Rennes. 


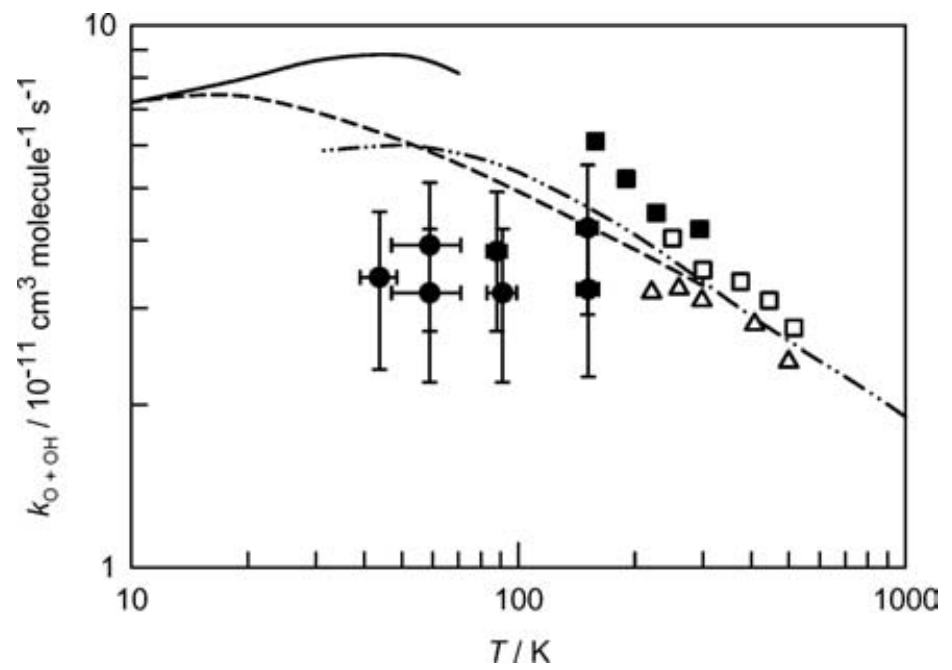

Figure 6. Comparison of temperature-dependent experimental and theoretical data for the reaction between $\mathrm{OH}$ radicals and $\mathrm{O}\left({ }^{3} \mathrm{P}\right)$ atoms. The present corrected values of $k$ are shown as closed filled circles. The corrected values of the rate constants are plotted at a temperature at the midpoint of the temperature range resulting from heating of the supersonic flow by laser absorption, with the horizontal lines through the points indicating the range of estimated temperatures. The vertical lines through the corrected data represent the range of the estimated uncertainties. The data of Howard \& Smith $(1980,1981)$ and Smith \& Stewart (1994) are represented by open and filled squares, respectively, and those of Lewis \& Watson (1980) by open triangles. The lines represent theoretical results: dashed - the recommended rate expression of Davidsson \& Stenholm (1990); solid - the quantum SACM calculations of Troe \& Ushakov (2001); dashed-dotted - the classical trajectory calculations of Troe \& Ushakov (2001).

In a significant breakthrough in Birmingham, a new co-photolysis method has been employed enabling the measurement of this rate constant down to around $40 \mathrm{~K}$. We have employed $157 \mathrm{~nm}$ radiation from an $\mathrm{F}_{2}$ excimer laser to produce oxygen atoms from the in-situ photolysis of $\mathrm{O}_{2}$ within the cold supersonic flow, while at the same time generating a much smaller concentration of $\mathrm{OH}$ radicals from the photolysis of $\mathrm{H}_{2} \mathrm{O}$. The oxygen atoms are initially formed in both the ground $\left({ }^{3} \mathrm{P}\right)$ and excited $\left({ }^{1} \mathrm{D}\right)$ states, but the use of $\mathrm{N}_{2}$ buffer gas ensures rapid relaxation to the ground state. The $\mathrm{OH}$ concentration was followed in the usual way by pulsed LIF detection. The oxygen atom concentration was determined by careful measurements of the photolysis laser fluence and $\mathrm{O}_{2}$ concentrations used, coupled with a knowledge of the absorption cross section at $157 \mathrm{~nm}$. An example of a typical decay trace obtained at $83 \mathrm{~K}$ is shown in Figure 5 . The results obtained (after correction for thermal expansion induced by the photolysis laser) are shown in Figure 6 . These results have been accepted for publication in a special issue of the Journal of Physical Chemistry A in honour of Jürgen Troe (Carty et al. 2005).

\subsection{Reaction Kinetics of the $C_{2}$ Radical}

The $\mathrm{C}_{2}$ molecule is found throughout the Universe. It has been observed in widely differing environments ranging from cold regions such as interstellar clouds (Cecchi-Pestellini \& Dalgarno 2002), circumstellar envelopes (Bakker et al. 1997) and comets (A'Hearn et al. 1995) to hot media such as hydrocarbon flames (Baronavski \& McDonald 1977), plasmas (Rennick et al. 2004) or stellar atmospheres (Brault et al. 1982). Understanding its chemistry and more specifically the temperature dependence of rate constants for reactions with other reagents is therefore of major interest. To date, most of the available 

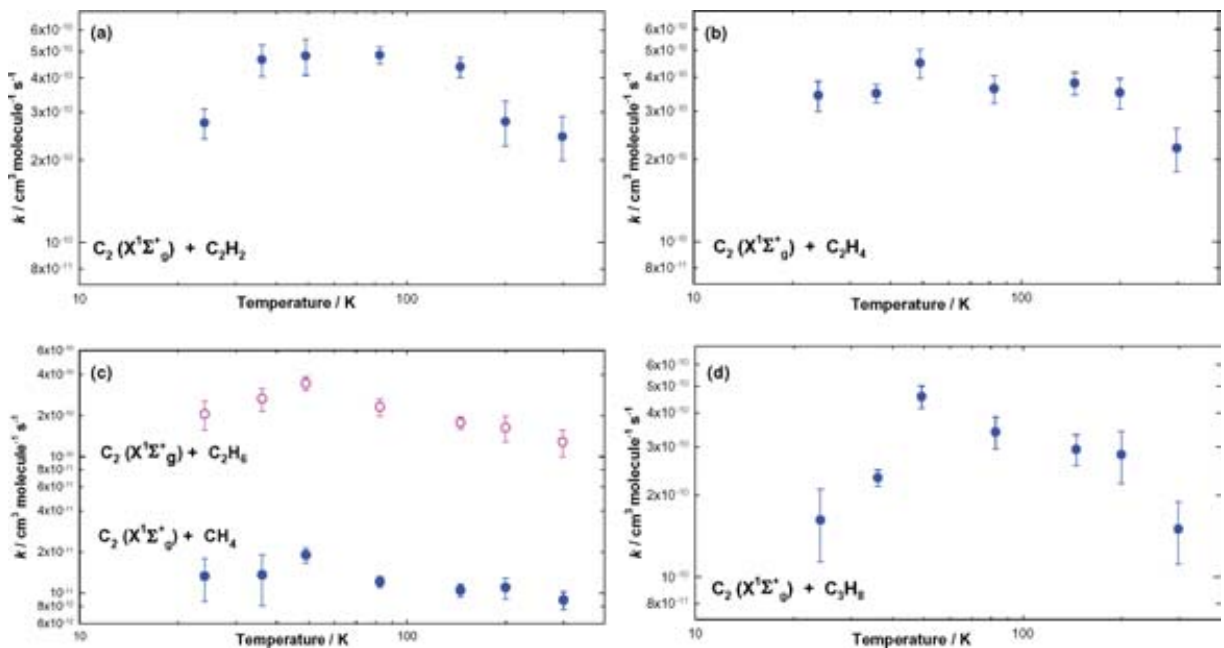

Figure 7. Rate constants for reaction of ${ }^{1} \mathrm{C}_{2}$ with various hydrocarbons displayed on a log-log scale as a function of temperature: $(a){ }^{1} \mathrm{C}_{2}+\mathrm{C}_{2} \mathrm{H}_{2} ;(b){ }^{1} \mathrm{C}_{2}+\mathrm{C}_{2} \mathrm{H}_{4} ;(c)$ open circles, ${ }^{1} \mathrm{C}_{2}+$ $\mathrm{C}_{2} \mathrm{H}_{6}$; closed circles, ${ }^{1} \mathrm{C}_{2}+\mathrm{CH}_{4} ;(d){ }^{1} \mathrm{C}_{2}+\mathrm{C}_{3} \mathrm{H}_{8}$.

experimental data, however, have been obtained at room temperature and essentially relate to the reactivity of $\mathrm{C}_{2}$ with hydrocarbons. While studies have also been carried out at higher temperatures, no measurements have been performed below $300 \mathrm{~K}$ in the gas phase.

The reaction kinetics of ground state $X^{1} \Sigma_{g}^{+} \mathrm{C}_{2}$ (hereafter ${ }^{1} \mathrm{C}_{2}$ ) are somewhat complicated by the presence of a low-lying triplet state $a^{3} \Pi_{u}$, (hereafter ${ }^{3} \mathrm{C}_{2}$ ) at only $610 \mathrm{~cm}^{-1}$ (i.e., $\sim 880 \mathrm{~K}$ ) above the ground state. In our experiments, both ${ }^{1} \mathrm{C}_{2}$ and ${ }^{3} \mathrm{C}_{2}$ are thought to be formed by the $193 \mathrm{~nm}$ laser photolysis of $\mathrm{C}_{2} \mathrm{Cl}_{4}$, and we have exploited this fact to study down to temperatures as low as $24 \mathrm{~K}$ the kinetics of both species with $\mathrm{O}_{2}$ and NO (to appear in a special issue of the Journal of Physical Chemistry A in honour of Jürgen Troe; Páramo et al. 2005a) as well as the hydrocarbons $\mathrm{CH}_{4}, \mathrm{C}_{2} \mathrm{H}_{2}, \mathrm{C}_{2} \mathrm{H}_{4}, \mathrm{C}_{2} \mathrm{H}_{6}$ and $\mathrm{C}_{3} \mathrm{H}_{8}$ (Páramo et al. 2005b). ${ }^{1} \mathrm{C}_{2}$ and ${ }^{3} \mathrm{C}_{2}$ were detected via LIF in the Mulliken $\left(D^{1} \Sigma_{u}^{+} \leftarrow X^{1} \Sigma_{g}^{+}\right)$and Swan $\left(d^{3} \Pi_{g} \leftarrow a^{3} \Pi_{u}\right)$ bands respectively. Further details are to be found in Páramo et al. (2005a,b).

${ }^{1} \mathrm{C}_{2}$ was found not to react with $\mathrm{O}_{2}$, while ${ }^{3} \mathrm{C}_{2}$ was rapidly quenched by $\mathrm{O}_{2}$, a fact which was exploited in subsequent measurements where $\mathrm{O}_{2}$ was added to the supersonic flow in order to quench (or equilibrate with ${ }^{1} \mathrm{C}_{2}$ ) the initially formed ${ }^{3} \mathrm{C}_{2}$. A selection of results for the reactions of ${ }^{1} \mathrm{C}_{2}$ with hydrocarbons is shown in Figure 7 .

As can be seen from Figure $7,{ }^{1} \mathrm{C}_{2}$ is an extremely reactive species not only at room temperature, but also down to extremely low temperatures. In the case of the reaction ${ }^{1} \mathrm{C}_{2}+\mathrm{C}_{2} \mathrm{H}_{4}$, the rate constant is found to be rapid and virtually temperature independent to $24 \mathrm{~K}$. Balucani et al. (2001) in a crossed molecular beam study found $\mathrm{C}_{4} \mathrm{H}_{3}+\mathrm{H}$ products at 15 and $29 \mathrm{~kJ} \mathrm{~mol}^{-1}$ collision energy (1200 and $2300 \mathrm{~K}$, respectively). Their $a b$ initio calculations predict a barrierless potential energy surface, in good agreement with the high value of the rate constant at low temperatures. For ${ }^{1} \mathrm{C}_{2}+\mathrm{C}_{2} \mathrm{H}_{2}$, similar behavior is observed, and Kaiser et al. (2003) found $\mathrm{C}_{4} \mathrm{H}+\mathrm{H}$ products at $24.1 \mathrm{~kJ} \mathrm{~mol}^{-1}$ collision energy $(2000 \mathrm{~K})$. Remarkably, ${ }^{1} \mathrm{C}_{2}$ also displays rapid rate constants with the saturated hydrocarbons $\mathrm{CH}_{4}, \mathrm{C}_{2} \mathrm{H}_{6}$ and $\mathrm{C}_{3} \mathrm{H}_{8}$. While the rate constant for reaction of ${ }^{1} \mathrm{C}_{2}$ with methane is an order of magnitude less than for the other hydrocarbons, it 
appears to show little or no temperature dependence down to the lowest temperatures, thus indicating a significant role for the ${ }^{1} \mathrm{C}_{2}$ species in methane-rich environments such as the atmosphere of Titan.

\subsection{Collisional Energy Transfer between Spin Orbit States of Atomic Carbon}

$\mathrm{C}\left({ }^{3} \mathrm{P}_{J}\right)$ is relatively abundant in interstellar space, and its intramultiplet transitions play an important role in cooling of interstellar environments. Energy transfer between these fine structure states is therefore of interest, particularly via collisions with $\mathrm{He}$ and $\mathrm{H}_{2}$, two of the most abundant interstellar collision partners.

$\mathrm{C}\left({ }^{3} \mathrm{P}_{J}\right)$ was generated by the $193 \mathrm{~nm}$ photolysis of $\mathrm{C}_{3} \mathrm{O}_{2}$, and detected by resonant vacuum-ultraviolet laser-induced fluorescence (VUV-LIF); that is, with both excitation and observation of the fluorescence on the $\left(2 \mathrm{~s}^{2} 2 \mathrm{p} 3 \mathrm{~s}^{3} \mathrm{P}_{J}-2 \mathrm{~s}^{2} 2 \mathrm{p}^{2}{ }^{3} \mathrm{P}_{J}\right)$ transition in atomic carbon. Pulses of VUV laser radiation were generated using four-wave mixing in Xe: dye laser radiation was tuned to $255.94 \mathrm{~nm}$ in order to excite Xe in a two-photon transition to its $5 \mathrm{p}^{5} 6 \mathrm{p}\left[2 \frac{1}{2}, 2\right]$ state and a second pulsed dye laser tuned through the wavelength range between 560 and $565 \mathrm{~nm}$ to generate VUV radiation between 165.9 and $165.4 \mathrm{~nm}$.

Following previous kinetic measurements made using direct VUV-LIF probing of $C\left({ }^{3} \mathrm{P}_{J}\right)$ in the CRESU, we were able to take advantage of the observation that the atomic carbon formed in the photolysis of $\mathrm{C}_{3} \mathrm{O}_{2}$ (and $\mathrm{CBr}_{4}$ for subsequent experiments) had a warm nascent distribution over spin orbit states to make measurements of the relaxation of each of these states in collisions with He at $15 \mathrm{~K}$. Fitting these relaxation data provided unique data on state-to-state rate coefficients for energy transfer between spin orbit states in $\mathrm{C}\left({ }^{3} \mathrm{P}_{J}\right)-\mathrm{He}$ collisions at $15 \mathrm{~K}$ (Le Picard et al. 2002).

While data on collisional energy transfer with $\mathrm{He}$ is of both fundamental and astrochemical interest, it is evident that the greatest astrochemical interest is in $\mathrm{H}_{2}$ as collision partner, as this is by far the majority species (by a factor of $10^{4}$ over CO) in dense ISCs. However, producing a pure $\mathrm{H}_{2}$ flow in the CRESU presents considerable difficulties. The combination of a large rotational energy gap spacing in molecular hydrogen, coupled with the restriction to $\Delta J= \pm 2$ arising from nuclear spin statistics, results in very slow rates of $\mathrm{H}_{2}-\mathrm{H}_{2}$ rotational energy transfer (RET). Normally in the CRESU expansion, RET occurs at a rate which maintains thermal equilibration during the passage through the Laval nozzle. In $\mathrm{H}_{2}$ this would not be the case. This would furthermore result in a ratio of specific heat capacities $\left(\gamma=C_{p} / C_{v}\right.$, a key quantity in aerodynamics) which would vary during the expansion, destroying the uniform flow.

In a major breakthrough, a working $\mathrm{H}_{2}$ Laval nozzle expansion was achieved by precooling the reservoir and nozzle with liquid nitrogen, allowing the $\mathrm{H}_{2}$ to relax into the two lowest rotational states before the expansion, at which point the gas behaves aerodynamically as a monatomic gas with a value of $\gamma$ of $5 / 3$, no further rotational relaxation being possible. A temperature profile of this flow obtained by impact pressure measurements is shown in Figure 8. Using this nozzle, we have been able to make preliminary measurements on spin orbit energy transfer in $\mathrm{C}\left({ }^{3} \mathrm{P}_{J}\right)-\mathrm{H}_{2}$ collisions at $7 \mathrm{~K}$, as well as (using $\mathrm{He}$ as carrier gas) extending our earlier $\mathrm{C}\left({ }^{3} \mathrm{P}_{J}\right)-\mathrm{He}$ measurements down to $7 \mathrm{~K}$. The results for $\mathrm{C}\left({ }^{3} \mathrm{P}_{J}\right)-\mathrm{H}_{2}$ collisions at $7 \mathrm{~K}$ are displayed in Figure 9, along with a comparison to the calculations of Schröder et al. (1991).

While agreement between experiment and theory is excellent for the rate constant for transfer from $J=1$ to $J=0$, the other rate constants measured are in strong disagreement with the theoretical results. A similar pattern is observed for $\mathrm{C}\left({ }^{3} \mathrm{P}_{J}\right)-\mathrm{He}$ collisions, and these systems are the subject of further detailed experimental investigation to confirm or otherwise this potentially very significant disagreement with theory at low temperatures. 


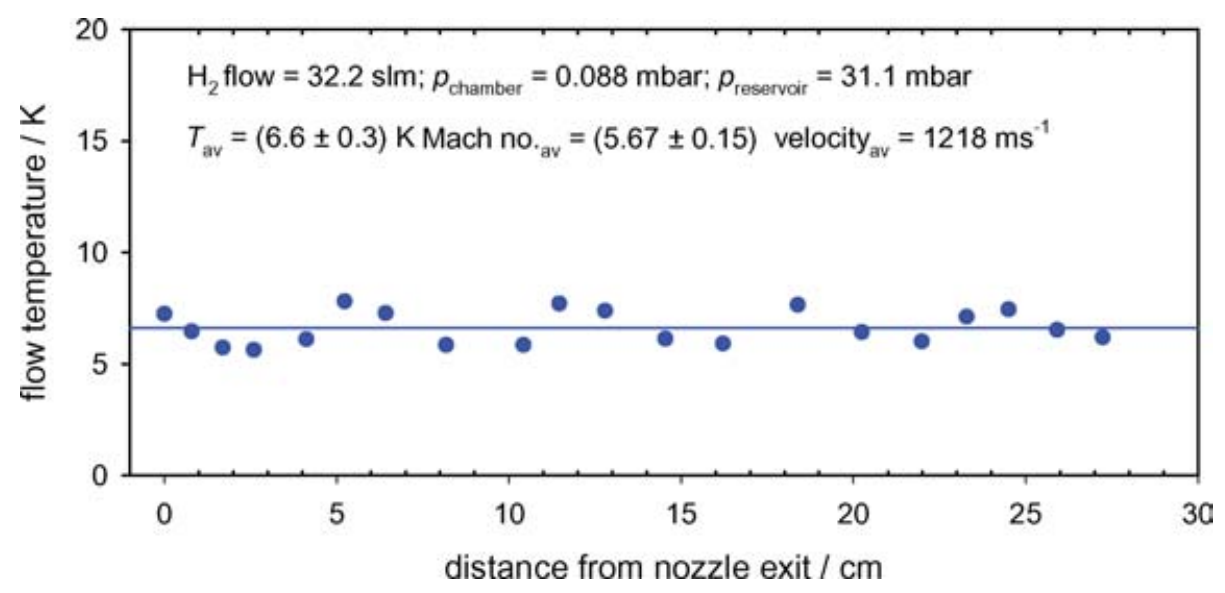

Figure 8. Temperature profile of new $\mathrm{H}_{2}$ Laval nozzle flow.
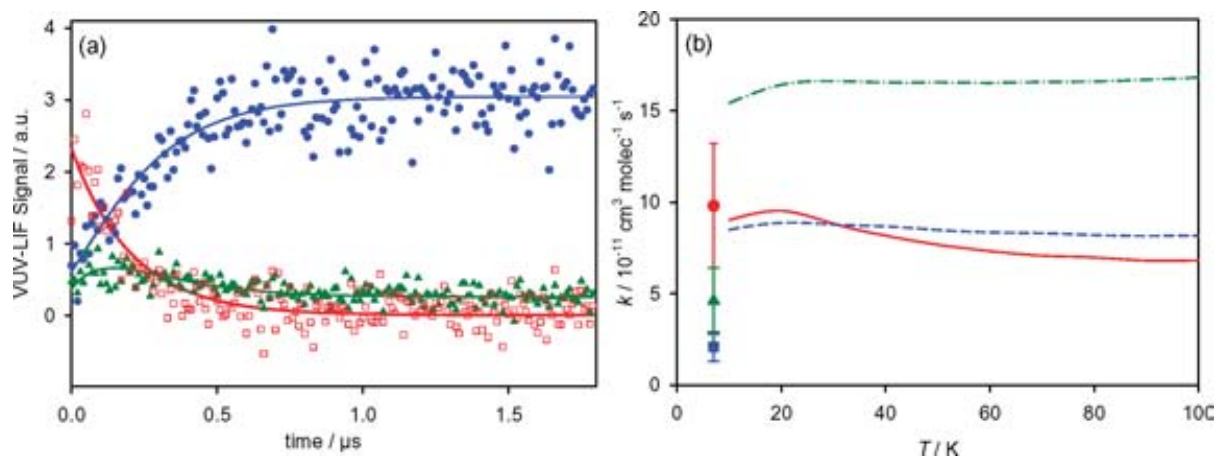

Figure 9. (a) VUV-LIF signal from $\mathrm{C}\left({ }^{3} \mathrm{P}_{J}\right)$ as a function of time delay after photolysis in a $7 \mathrm{~K} \mathrm{H}_{2}$ buffer. The closed circles show relaxation of the $J=0$ state, the filled triangles relaxation of the $J=1$ state, and the open squares relaxation of the $J=2$ state. The solid lines indicate a simultaneous fit to these data, yielding the experimental points shown to the right; (b) experimental intramultiplet state-to-state rate constants for $\left.\mathrm{C}^{3} \mathrm{P}_{J}\right)$ in collision with normal $\mathrm{H}_{2}$ at $7 \mathrm{~K}$ (solid symbols), along with the calculations (lines) of Schröder et al. (1991). The circle and solid line refer to transfer from $J=1$ to $J=0$, the square and dashed line to transfer from $J=2$ to $J=0$, and the triangle and dashed-dotted line to transfer from $J=2$ to $J=1$.

\subsection{Reaction Kinetics of the $\mathrm{C}_{4} \mathrm{H}$ Radical}

Work is currently underway to elucidate the reaction kinetics of the $\mathrm{C}_{4} \mathrm{H}$ radical, which has been detected in both interstellar clouds (Olano et al. 1988) and circumstellar envelopes (Dayal \& Bieging 1993), and is implicated in the synthesis of complex hydrocarbons in the chemistry of planets such as Titan (Zwier \& Allen 1996). To our knowledge there have been no kinetic studies to date of $\mathrm{C}_{4} \mathrm{H}$ reactions at any temperature. In our experiments, $\mathrm{C}_{4} \mathrm{H}$ is formed by $193 \mathrm{~nm}$ excimer laser photolysis of diacetylene, $\mathrm{C}_{4} \mathrm{H}_{2}$, and detected by laser induced fluorescence around $415 \mathrm{~nm}$ (Hoshina et al. 1998). Preliminary results have been obtained at $52 \mathrm{~K}$ for reaction of $\mathrm{C}_{4} \mathrm{H}$ with $\mathrm{C}_{2} \mathrm{H}_{2}, \mathrm{C}_{2} \mathrm{H}_{6}, \mathrm{C}_{3} \mathrm{H}_{8}$ which all show fast rate constants in the range $1.54 \times 10^{-10} \mathrm{~cm}^{3}$ molecule $\mathrm{e}^{-1} \mathrm{~s}^{-1}$. The reaction of $\mathrm{C}_{4} \mathrm{H}$ with $\mathrm{CH}_{4}$ at $52 \mathrm{~K}$ is too slow for us to measure $\left(k<10^{12} \mathrm{~cm}^{3}\right.$ molecule $\left.{ }^{-1} \mathrm{~s}^{-1}\right)$. We propose that likely products of the reactions of the $\mathrm{C}_{4} \mathrm{H}$ radical with unsaturated 
hydrocarbons are long carbon chains, e.g.,

$$
\mathrm{C}_{4} \mathrm{H}+\mathrm{C}_{2} \mathrm{H}_{2} \rightarrow \mathrm{HCCCCCCH}+\mathrm{H}
$$

Further measurements are underway at different temperatures and with a range of coreagents.

\section{Acknowledgements}

I would like to express my thanks to all my co-workers, in particular Ian W.M. Smith and Bertrand Rowe, who have been strongly involved in the development and exploitation of the CRESU technique. For the recent work described in this review I am grateful to my co-workers Coralie Berteloite, André Canosa, David Carty, Andrew Goddard, Sven Köhler, Alejandra Páramo, Sébastien Le Picard, Daniel Travers and Ian W.M. Smith. I thank the numerous agencies that have funded the work presented here including the UK EPSRC, the French national programmes PCMI and PNP, the European Commission, the Region of Brittany and Rennes Metropole. I am also very grateful to the European Commission for the award of a Marie Curie Chair for part of the period during which this work was performed under contract MEXC-CT-2004-006734.

\section{References}

A'Hearn, M.F., Millis, R.L., Schleicher, D.G., Osip, D.J., \& Birch, P.V. 1995, Icarus 118, 223

Bakker, E.J., van Dishoeck, E.F., Waters, L., \& Schoenmaker, T. 1997, A\&A A 323, 469

Balucani, N., Mebel, A.M., Lee, Y.T., \& Kaiser, R.I. 2001, J. Phys. Chem. A 105, 9813

Baronavski, A.P. \& McDonald, J.R. 1977, J. Chem. Phys. 66, 3300

Bocherel, P., Herbert, L.B., Rowe, B.R., Sims, I.R., Smith, I.W.M., \& Travers, D. 1996, J. Phys. Chem. 100, 3063

Brault, J.W., Delbouille, L., Grevesse, N., Roland, G., Sauval, A.J., \& Testerman, L. 1982, A\& A 108,201

Canosa, A., Sims, I.R., Travers, D., Smith, I.W.M., \& Rowe, B.R. 1997, A\& A 323, 644

Carty, D., Goddard, A., Khler, S., Sims, I.R., \& Smith, I.W.M. 2005, J. Phys. Chem. A, in press

Cecchi-Pestellini, C. \& Dalgarno, A. 2002, MNRAS 331, L31

Chastaing, D., James, P.L., Sims, I.R., \& Smith, I.W.M. 1998, Faraday Discuss. 165

Chastaing, D., Le Picard, S.D., \& Sims, I.R. 2000, J. Chem. Phys. 112, 8466

Chastaing, D., Le Picard, S.D., Sims, I.R., \& Smith, I.W.M. 2001, A\&3A 365, 241

Davidsson, J. \& Stenholm, L.G. 1990, A\&A 230, 504.

Dayal, A. \& Bieging, J.H. 1993, Astrophys. J. 407, L37

Herbst, E. 2001, Chem. Soc. Rev. 30, 168

Herbst, E., Lee, H.H., Howe, D.A., \& Millar, T.J. 1994, MNRAS 268, 335

Hoshina, K., Kohguchi, H., Ohshima, Y., \& Endo, Y. 1998, J. Chem. Phys. 108, 3465

Howard, M.J. \& Smith, I.W.M. 1980, Chem. Phys. Lett. 69, 40

Howard, M.J. \& Smith, I.W.M. 1981, J. Chem. Soc.-Faraday Trans. II 77, 997

James, P.L., Sims, I.R., Smith, I.W.M., Alexander, M.H., \& Yang, M.B. 1998, J. Chem. Phys. 109, 3882

Kaiser, R.I., Balucani, N., Charkin, D.O., \& Mebel, A.M. 2003, Chem. Phys. Lett. 382, 112

Le Picard, S.D., Honvault, P., Bussery-Honvault, B., Canosa, A., Laube, S., Launay, J.M., Rowe, B., Chastaing, D., \& Sims, I.R. 2002, J. Chem. Phys. 117, 10109

Lewis, R.S. \& Watson, R.T. 1980, J. Phys. Chem. 84, 3495

Olano, C.A., Walmsley, C.M., \& Wilson, T.L. 1988, A\&A 196, 194

Páramo, A., Canosa, A., Le Picard, S.D., \& Sims, I.R. 2005a, J. Phys. Chem. A, in press

Páramo, A., Canosa, A., Le Picard, S.D., \& Sims, I.R. 2005b, in preparation

Rennick, C.J., Smith, J.A., Ashfold, M.N.R., \& Orr-Ewing, A.J. 2004, Chem. Phys. Lett. 383, 518

Rowe, B.R., Dupeyrat, G., Marquette, J.B., \& Gaucherel, P. 1984, J. Chem. Phys. 80, 4915 
Rowe, B.R. \& Marquette, J.B. 1987, Int. J. Mass Spectrom. Ion Proc. 80, 239

Schröder, K., Staemmler, V., Smith, M.D., Flower, D.R., \& Jaquet, R. 1991, J. Phys. B-At. Mol. Opt. Phys. 24, 2487

Sims, I.R. 1997, Research in Chemical Kinetics, eds. R.G. Compton, \& G. Hancock (Oxford: Blackwell Science), vol. 4, pp. 121

Sims, I.R., Queffelec, J.L., Defrance, A., Rebrionrowe, C., Travers, D., Bocherel, P., Rowe, B.R., \& Smith, I.W.M. 1994, J. Chem. Phys. 100, 4229

Sims, I.R., Queffelec, J.L., Travers, D., Rowe, B.R., Herbert, L.B., Karthauser, J., \& Smith, I.W.M. 1993, Chem. Phys. Lett. 211, 461

Sims, I.R. \& Smith, I.W.M. 1995, Annu. Rev. Phys. Chem. 46, 109

Smith, I.W.M. \& Rowe, B.R. 2000, Acc. Chem. Res. 33, 261

Smith, I.W.M. \& Stewart, D.W.A. 1994, J. Chem. Soc. Faraday Trans. 90, 3221

Terzieva, R. \& Herbst, E. 1998, Ap. J. 501, 207

Troe, J. \& Ushakov, V.G. 2001, J. Chem. Phys. 115, 3621

Woon, D.E. \& Herbst, E. 1997, Ap. J. 477, 204

Zwier, T.S. \& Allen, M. 1996, Icarus 123, 578

\section{Discussion}

DAlgarno: Comment: I have calculations that would enable you to determine whether or not your measurements are correct (Krems et al. 2002; Phys. Rev. A 66, 030702).

Sims: We would be pleased to check your calculations against our experimental data. However, as the paper referred to only contains calculations for $\mathrm{C}\left({ }^{3} \mathrm{P}_{J}\right)+{ }^{3} \mathrm{He}$ at temperatures below $1 \mathrm{~K}$, further calculations would be necessary to enable the comparison, and these we would welcome.

BLACK: In the old combustion literature there was a hint that $\mathrm{C}_{2}+\mathrm{H}_{2} \rightarrow \mathrm{C}_{2} \mathrm{H}+\mathrm{H}$ goes at a rate coefficient sufficient to be astrophysically interesting. Can this be measured in your experiment?

Canosa: The $\mathrm{C}_{2}+\mathrm{H}_{2}$ reaction was studied by Pitts et al. (1982; Chem. Phys. 68, 417) within $300 \mathrm{~K}$ and $500 \mathrm{~K}$. They found a significant rate at high temperatures $\left(7.9 \times 10^{-12}\right.$ $\mathrm{cm}^{3} \mathrm{~s}^{-1}$ molecule ${ }^{-1}$ ), but the rate falls to $1.38 \times 10^{-12} \mathrm{~cm}^{3} \mathrm{~s}^{-1}$ molecule ${ }^{-1}$ at room temperature. Such a behavior is not favorable to get a measurable rate coefficient at lower temperatures using our technique. Therefore we did not consider this system. 